\title{
The Transition in the Two-Dimensional Step Model: A Kosterlitz-Thouless Transition in Disguise
}

\author{
Peter Olsson and Petter Holme \\ Department of Theoretical Physics, Umeå University, 90187 Umeå, Sweden
}

(November 10, 2018)

\begin{abstract}
Evidence for a Kosterlitz-Thouless transition in the 2D step model is obtained from Monte Carlo determinations of the helicity modulus. It is argued that the free energy of a single vortex at the center of the system depends logarithmically on the system size in spite of the fact that the spin interaction is not harmonic for small differences in the spin angles. We conclude that this is the reason for the Kosterlitz-Thouless transition in the 2D step model and that the harmonic spin interaction not is a necessary requirement.
\end{abstract}

05.50.+q, 05.70.Jk, 64.60.Cn, 64.70.-p

The phase transition in two-dimensional (2D) $\mathrm{XY}$ models is known to take place through the vortex unbinding mechanism due to Kosterlitz and Thouless (KT) [1]. From the principles of universality one expects this transition to remain the same independent of details of the system as e.g. the underlying lattice structure. The precise spin interaction potential $U(\phi)$, where $\phi$ is the angle difference between neighboring spins, is not supposed to be essential either. $U(\phi)$ is however required to be periodic in $2 \pi$ and it seems always to have been presumed that the interaction in addition has to be harmonic for small $\phi$. The harmonicity for small $\phi$ has to do with the energetics for vortex formation. With a harmonic potential the energy for a single vortex in a $L \times L$ lattice goes as $\ln L$, and in the classical argument by Kosterlitz and Thouless this property is crucial for the transition.

The subject of the present Letter is the $2 \mathrm{D}$ step model which is an XY model with a spin interaction that has no harmonic component. $U(\phi)$ is instead a step-like function

$$
U(\phi)=-J \operatorname{sign}(\cos \phi)
$$

Since this potential is flat around $\phi=0$ there is no $\ln L$ dependence of the energy for a single vortex. This energy is instead independent of system size and one would therefore expect a non-vanishing density of free vortices at all finite temperatures, and consequently no phase transition. Against that background the evidence from simulations for a transition were very intriguing. The first clear evidence for a transition was obtained from a Monte Carlo (MC) study of the susceptibility and the specific heat [2]. The increase of the susceptibility with lattice size was considered to suggest a phase transition at $T \approx 1$.1. Later simulations also provided evidence that the transition actually is in the same universality class as the harmonic XY models [3]. The similarity of the behavior close to the transition of an harmonic XY model and the step model also led these authors to question the vortex unbinding as the mechanism behind the KT transition [3]. How could a transition driven by vortices be altogether insensitive to the very different energy cost for vortices in the two models?
In the present Letter we address the question of the necessity of an harmonic spin potential for the KT transition by examining the behavior of the $2 \mathrm{D}$ step model. We first calculate the helicity modulus and show that the behavior of this quantity gives strong support for a KT transition. We then demonstrate that the cost in free energy for a single vortex at the center of the system in fact goes as $\ln L$. It is this feature that stabilizes the low temperature phase. Finally, we refine the arguments to obtain quantitatively satisfactory estimates.

The helicity modulus $\Upsilon$, is a convenient quantity for the study of KT transitions due to its universal value $2 T / \pi$ at the transition [4. approach to this universal value with $L[6]$. The usual procedure in MC simulations is to determine $\Upsilon$ from a correlation function which involves some derivatives of $U(\phi)$ [7]. Clearly, with a step-like potential the derivatives of the potential cannot be calculated and this expression cannot be used. A way out is to instead start from the defining expression for the helicity modulus

$$
\Upsilon=\left.\frac{\partial^{2} F}{\partial \Delta^{2}}\right|_{\Delta=0},
$$

and perform the simulations with fluctuating twist boundary conditions [8]. In these simulations one collects a histogram of the total twist $P(\Delta)$. Since the probability for a certain twist is related to the free energy through $P(\Delta) \propto e^{-F(\Delta) / T}$ Eq. (2) becomes

$$
\Upsilon=-\left.T \frac{\partial^{2} \ln P}{\partial \Delta^{2}}\right|_{\Delta=0} .
$$

The simulations are done with twist variables in the two directions, $\Delta_{x}$ and $\Delta_{y}$, which beside the spin variables $\theta_{i}$ are updated with the Metropolis algorithm. With $\mathbf{r}_{i j}$ a unit vector between nearest neighbors and $\boldsymbol{\Delta}=\left(\Delta_{x}, \Delta_{y}\right)$, the Hamiltonian may be written

$$
H=\sum_{\langle i j\rangle} U\left(\theta_{i}-\theta_{j}-\frac{1}{L} \mathbf{r}_{i j} \cdot \boldsymbol{\Delta}\right)=\sum_{\langle i j\rangle} U\left(\phi_{i j}\right) .
$$

To get a good acceptance ratio for the twist update it is necessary to make use of $L$ different twist variables 
in each direction, with $\Delta_{x}=\sum_{k=1}^{L} \Delta_{x}^{(k)}$, (and similarly in the $y$ direction) where $k$ is the column (row) number. In our simulations, which for convenience were for a $O(256)$ model, we used the potential $U(\phi)=-J$ for 129 angle differences $[-\pi / 2, \pi / 2]$ and $+J$ for the remaining 127 [9]. This choice is not expected to be important for the transition properties, but gives a slight shift of the transition temperature as compared to the potential of Eq. (1). The length of the runs were typically $5 \times 10^{8} / L$ sweeps through the lattice.

In Fig. 1] we show the histogram $P(\Delta)$ from Monte Carlo simulations at $T=0.05 \mathrm{~J}$. Since the histogram is peaked around $\Delta=0$ the figure immediately gives evidence for a low temperature phase with a finite stiffness. To determine $\Upsilon$ we fit a quadratic curve to $\ln P$ for $|\Delta / \pi|<1 / 3$ and obtain $\Upsilon / T=0.789$ (4) (where the given error is one standard deviation). Note that this is slightly larger than the universal value $2 / \pi \approx 0.637$, which is required for a stable low temperature phase.

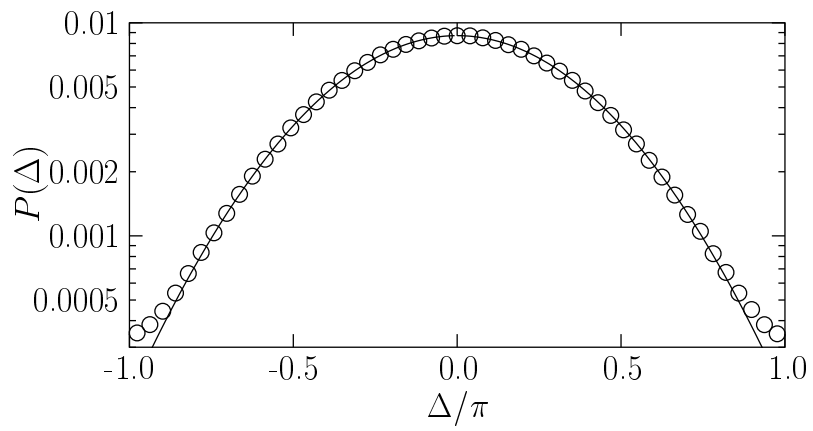

FIG. 1. Twist histogram obtained through MC simulations at $T=0.05$. The solid line is obtained by fitting the data to a quadratic form which gives $\Upsilon / T=0.789$.

An important feature of the step model is the gap in excitation energies; there are no excitations with energy $<2 J$. At $T \ll J$ the system is therefore at all times in one of its numerous ground states which means that the histogram $P(\Delta)$ is independent of temperature. From Eq. (3) then follows a linear temperature dependence for $\Upsilon$. This is in contrast to harmonic XY models for which $\Upsilon / J$ approaches unity in the low-temperature limit.

The temperature dependence of $\Upsilon$ is shown in Fig. 2 for several system sizes together with the dashed line for the universal jump condition $2 T / \pi$. We note that the curves start out linearly at low temperatures, become size-dependent at $T / J \approx 0.75$, cross the universal line and then drop down to zero. Beside the unusual linear temperature-dependence at low $T$ this behavior is just as in an ordinary harmonic XY model and therefore precisely what one would expect for a KT transition.

To determine the KT temperature we make use of the finite size dependence of $\Upsilon$ 6]. We follow the procedure in Ref. [10] of first fitting our MC data for $\Upsilon$ from a narrow temperature interval to second order polynomials in $T$, one for each $L$, and then fit the data to the expression

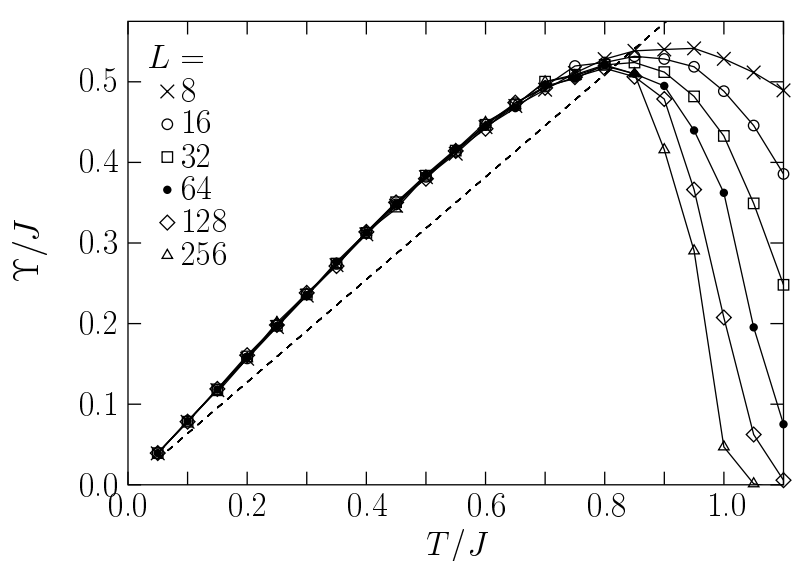

FIG. 2. The helicity modulus versus temperature for $L=8$ through 256. The rapid decrease down to zero directly follows the crossing of the data with the universal line, $2 T / \pi$ (dashed line). This behavior is suggestive of a KT transition. Note that even though $\Upsilon \rightarrow 0$ at low temperatures, the system remains in the low temperature phase since $\Upsilon / T>2 / \pi$.

$$
\Upsilon_{L}\left(T_{\mathrm{KT}}\right)=\frac{2 T_{\mathrm{KT}}}{\pi}\left(1+\frac{1}{A+2 \ln L}\right) .
$$

which amounts to adjusting $T_{\mathrm{KT}}$ and $A$ to get the best possible fit. Using data for $L \geq 16$ we obtained $T_{\mathrm{KT}}=$ $0.765(6)$. Fig. 3 illustrates the good fit of the data at the transition temperature to the line from Eq. (化). We consider the above MC data to be strong evidence for a KT transition. This is in agreement with the conclusion in Ref. [3] that the step model is in the same universality class as the harmonic XY model.

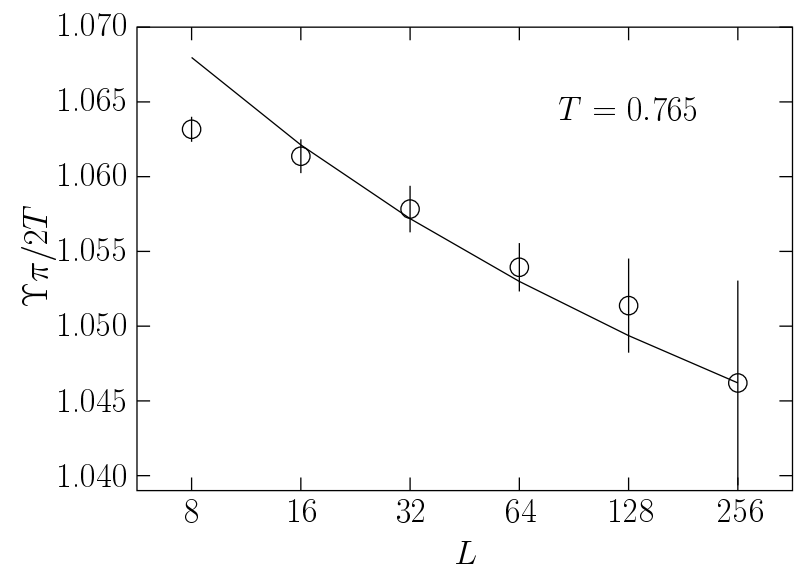

FIG. 3. Determination of the transition temperature. The solid line is Eq. (4) and the data points are the helicity modulus from our simulations.

We now propose an analytical analysis in order to understand this behavior. We focus on the properties in the low temperature phase where the angular differences are restricted to the low energy region, $|\phi| \leq \pi / 2$. A central idea in the present letter is to note that, while in the harmonic XY model the spinwave-vortex interaction only is a smooth perturbation, in the step model this interaction leads to a dramatic and crucial effect: while the energy of a system with a single vortex fixed in the center of the 
system is finite, the free energy of this system grows as $\ln L$. This is due to the change in entropy of spinwave fluctuations for the configuration with the fixed vortex, as compared to the vortex free case. Note that this is the entropy associated with a fixed vortex, not the positional entropy associated with a free vortex's variable location.

To demonstrate the existence of this spinwave entropy we consider the configuration of spins in Fig. 位 where we slightly reorganize the spins and delete the links in the radial direction. We will return below to the approximation involved in this step.
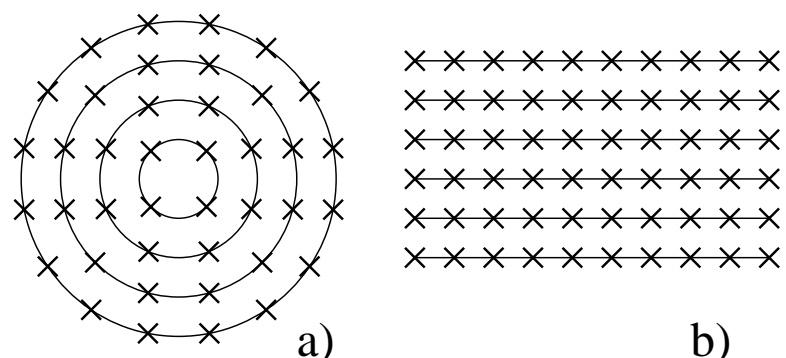

a)

b)

FIG. 4. Arrangement of links used to a) argue for the change in spinwave entropy for a vortex at a fixed position and b) estimate the helicity modulus.

The condition for having a positive vortex in the center of the system in Fig. A $\mathrm{a} a$ is that the phase rotates by $2 \pi$ along each of the circles. We therefore introduce $\Phi_{r}=$ $\sum \phi$ along the circle with radius $r$, which is a sum of $\approx$ $2 \pi r$ values and let $\Omega_{r}\left(\Phi_{r}\right)$ denote the number of possible combinations of the $\phi$ :s at distance $r$ as a function of $\Phi_{r}$, which is defined only for $\Phi_{r}=2 \pi n$ (with integer $n$ ). The probability for having a (positive) vortex is then determined by the product

$$
P_{\mathrm{vort}} \approx \prod_{r=1}^{L} \frac{\Omega_{r}(2 \pi)}{\Omega_{r}(0)}
$$

The fraction $\Omega_{r}(2 \pi) / \Omega_{r}(0)$ may be calculated if we temporarily open up a closed path that makes up a circle. Since we want the same number of links along this path we need one more spin variable at one of the endpoints, which we take to be independent of the other endpoint. $\Phi_{r}$ then becomes a sum of $\approx 2 \pi r$ independent variables $\phi$. With the average of $\phi$ being equal to zero and its variance given by $\sigma^{2}$ the distribution of $\Phi_{r}$ for this open path becomes a Gaussian with width $2 \pi r \sigma^{2}$ :

$$
\Omega_{r}^{\text {open }}\left(\Phi_{r}\right) \propto \exp \left(-\frac{\Phi_{r}^{2}}{2 \pi r \times 2 \sigma^{2}}\right)
$$

We now make use of the fact that the number of possible configurations for the open and closed paths are the same if the spins at the endpoints of the open path are equal. Since this condition is equal to having $\Phi_{r}=2 \pi n$ we conclude that $\Omega_{r}(2 \pi n)=\Omega_{r}^{\text {open }}(2 \pi n)$. From Eqs. (5) and (6) the probability for a vortex then becomes

$$
P_{\text {vort }}=\exp \left(-\frac{\pi}{\sigma^{2}} \sum_{r=1}^{L} \frac{1}{r}\right) \approx \exp \left(-\frac{\pi}{\sigma^{2}} \ln L\right),
$$

for the entropy of a fixed vortex we obtain $S_{\text {vort }}=$ $\ln P_{\mathrm{vort}}=-\frac{\pi}{\sigma^{2}} \ln L$, and the free energy for a vortex at a fixed position in a system of size $L$ finally becomes

$$
F_{\text {vort }}=-T S_{\text {vort }}=T \frac{\pi}{\sigma^{2}} \ln L .
$$

For the harmonic XY model the well-known argument for the phase transition gives the free energy for having a vortex at any of the $L^{2}$ positions as

$$
\Delta F=(\pi J-2 T) \ln L,
$$

and the transition takes place at $T / J=2 / \pi$. In the step model the corresponding expression becomes

$$
\Delta F=F_{\text {vort }}-2 T \ln L=T\left(\frac{\pi}{\sigma^{2}}-2\right) \ln L,
$$

where the temperature, at first sight only appears as a prefactor. However, there is a hidden temperature dependence in the variance $\sigma^{2}$. At low enough temperatures the $\phi_{i j}$ are restricted to the interval $[-\pi / 2, \pi / 2]$ but with increasing temperature the $\phi_{i j}$ will more often take values outside this interval, and $\sigma^{2}$ will increase. Therefore, if $\Delta F$ is positive at low temperatures it will turn negative at some finite temperature and this will give the transition. But if $\sigma^{2}>\pi / 2$ already at low temperatures there will be no transition.

To see how the local restrictions on the angle differences give rise to the non-zero helicity modulus we now turn to a rectangular geometry and consider the determination of $\Upsilon$ from the distribution of $\Delta_{x}$ and $\Delta_{y}$. One point with examining the distribution of the twist is to give predictions that are easy to compare with $\mathrm{MC}$ simulations. In the simplest approximation we again delete all links in the perpendicular direction, as in Fig. đfb. For a single row the number of configurations consistent with a certain total twist becomes $\Omega_{\text {row }}(\Delta) \propto \exp \left(-\Delta^{2} / 2 L \sigma^{2}\right)$, in analogy with Eq. (6), and the number of configurations for the whole system with $L$ rows becomes

$$
\Omega(\Delta)=\left[\Omega_{\text {row }}(\Delta)\right]^{L} \propto \exp \left(-\Delta^{2} / 2 \sigma^{2}\right) .
$$

For the free energy we then arrive at $F(\Delta)=T \Delta^{2} / 2 \sigma^{2}$ and with Eq. (2) the helicity modulus becomes

$$
\Upsilon=T / \sigma^{2} .
$$

In the absence of perpendicular links and at low temperatures, the $\phi_{i j}$ have a rectangular distribution, and from elementary integrals one finds $\sigma^{2}=\pi^{2} / 12 \approx 0.822$. Through Eq. (8) this gives $\Upsilon / T \approx 1.22$ which is about $50 \%$ larger than $\Upsilon / T \approx 0.789$ from Monte Carlo simulations, cf. Fig. 1. A better estimate will be obtained below by including some links in the perpendicular direction.

Comparing Eqs. (7) and (8) we find $F_{\text {vort }}(L)=\Upsilon \pi \ln L$ which is the same relation as in the harmonic model. This 
shows that our two different calculations are equivalent which is a consequence of using the same approximation in both cases, i.e. neglecting all links perpendicular to the direction of interest.

We now discuss the assumption used above, namely that the qualitatively correct behavior in a certain direction may be obtained even though one neglects the perpendicular links. This assumption is true only if the relative reduction of the number of allowed configurations obtained by introducing perpendicular links is essentially independent of the total twist in the direction of interest. We argue that this is a plausible assumption by considering two sets of configurations: 1) the set of all twist free configurations and 2) the set of configurations with a twist $\Delta_{x}$. There is then a transformation $\phi_{i j}+\Delta / L \rightarrow \phi_{i j}$ on all the horizontal links that transforms each member in the twist free set into a corresponding one in the twisted set. Since this transformation not affects the angle difference at the perpendicular links, it follows that the effect of the perpendicular links will be to exclude the same number of configurations in both these sets and this suggests that the relative reduction due to the perpendicular links will be independent of $\Delta$. However, this argument only serves to make our assumption a reasonable one; it is not conclusive. There is nothing that guarantees that the relative reduction of the allowed configurations (with $\left|\phi_{i j}\right|<\pi / 2$ for all horizontal links) will be the same for the two different sets. That a certain member of the twist free set is allowed doesn't imply that the corresponding member in the twisted set is allowed too.

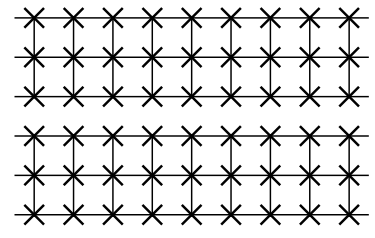

a)

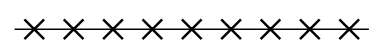

$x \times x \times x \times x$

b)
FIG. 5. The configuration of links used to get quantitatively reasonable values. The starting point is given in panel a) and after integrating out the upper and lower rows on each triple row one obtains the links shown in panel b).

Even though we have argued that the perpendicular links may be neglected in a qualitative discussion, they have to be included in order to get reasonable quantitative estimates, since they do affect the variance $\sigma^{2}$. To include some perpendicular links we consider the configuration in Fig. Fa, where the perpendicular links have been deleted at every third row only. The approach is then to integrate out the upper and lower rows of spins in each of these triple rows to give $L / 3$ one-dimensional rows, cf. Fig. Eb. These integrations may be done analytically or by Monte Carlo simulations on that geometry. The integrations also give correlations to the neighboring and next neighboring links, $\left\langle\phi \phi^{\prime}\right\rangle$ and $\left\langle\phi \phi^{\prime \prime}\right\rangle$ (cf. Fig. 6), which together give an effective variance $\sigma_{\text {eff }}^{2}$ :

$$
\sigma_{\text {eff }}^{2}=\frac{1}{L}\left\langle\left(\sum_{i=1}^{L} \phi_{i}\right)^{2}\right\rangle \approx \sigma^{2}+\left\langle\phi \phi^{\prime}\right\rangle+\left\langle\phi \phi^{\prime \prime}\right\rangle .
$$

With $L / 3$ rows, as in Fig. 5 5 , the expression for the helicity modulus becomes $\Upsilon=T /\left(3 \sigma_{\text {eff }}^{2}\right)$. From our MC simulations on the geometry of Fig. 5a we get $\sigma^{2}=0.672$, $\left\langle\phi \phi^{\prime}\right\rangle=-0.120$, and $\left\langle\phi \phi^{\prime \prime}\right\rangle=-0.026$. The first two of these numbers are easily obtained by integrating analytically with symbolic software. With Eq. (9) this gives $\sigma_{\text {eff }}^{2}=0.526$ and $\Upsilon / T=0.633$ which is less than $20 \%$ off $\Upsilon / T=0.789$ obtained above.

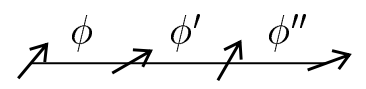

FIG. 6. Illustration to the link-link correlations in Eq. (9).

To conclude we have presented evidence from simulations that the 2D step model actually undergoes a KT transition. We have argued that the reason for the stability of the low temperature phase against the formation of free vortices is the $\ln L$-dependence of the free energy for a vortex at a fixed position. From these results we are led to the conclusion that the harmonic spin interaction is not a necessary condition for a $\mathrm{KT}$ transition in a $2 \mathrm{D}$ spin model, and that the KT transition is a more general phenomenon than has so far been recognized.

The authors thank Prof. P. Minnhagen and Prof. S. Teitel for critical reading of the manuscript. Financial support from the Swedish Natural Science Research Council through Contract No. E-EG 10376-312 is gratefully acknowledged.

[1] J. M. Kosterlitz and D. J. Thouless, J. Phys. C 6, 1181 (1973).

[2] E. Sánchez-Velasco and P. Wills, Phys. Rev. B 37, 406 (1988).

[3] A. C. Irving and R. Kenna, Phys. Rev. B 53, 11568 (1996). R. Kenna and A. C. Irving, Nucl. Phys. B 485, 583 (1997).

[4] D. R. Nelson and J. M. Kosterlitz, Phys. Rev. Lett. 39, 1201 (1977).

[5] P. Minnhagen and G. G. Warren, Phys. Rev. B 24, 2526 (1981).

[6] H. Weber and P. Minnhagen, Phys. Rev. B 37, 5986 (1988).

[7] T. Ohta and D. Jasnow, Phys. Rev. B 20, 139 (1979).

[8] P. Olsson, Phys. Rev. Lett. 73, 3339 (1994); P. Olsson, Phys. Rev. B 52, 4511 (1995).

[9] This odd number comes from the desire to have a symmetric $U(\phi)$, together with one of the discrete $\phi$ being equal to zero.

[10] P. Olsson, Phys. Rev. B 52, 4526 (1995). 